\title{
PARASTTOSIS INTESTINAL EN LA PROVINCIA DE BOLIVAR
}

\author{
Dr. ANGEL TSCORZA
}

Hospitrl Ctuit, Guarerted

Lus parasitaris intestinales, a pesar de los pirgnesos terapcuticus, siguen siendo un irrporiante problema médico-sumitario, en la Provineia te Buliver. Sia frecuencia, en la práctica móditua, es elevada. Tisto se debe a que nu se ha intentudo una compaña sistemátitea en toda la región o enl todo el pais, somenyando por nujorar est estatlo thugićtico de las poblaciones, pruveyéndolas de agua verdaderamente potrible, etw.

\section{MATERLAL DH ESTUUIO}

Se estudiaron 583 pacientes, la mayoría, en el Hospital Civil de Guarinda, $y$ algumos, en corsulta privadr. La nyyuría de pacientes provienen do niveles econónicos bajisi, y por constguiente, de cacusus hábitos higiénictss. Finerom examinados elínicamente o inn vestigada la presencia de parásitos o sus huevori, en las heces.

E1 examer clinico y lin historia patológice, nos permitió reconocer ciortas caracteristicas o sintonı as más co-
Munos, Ios unismos que serán analizadesi a continuación.

\section{RESLLTADOS}

Subie el tolal de 583 pacientes, se encountró que 484 , es deeir, el $87,5 \%$ estaban parasitados. Isa frecuencia de mono o poliparasitosis, se presenta en la Tublá $\mathbf{I}$.

En la Tabla II se presenta la frecuen-

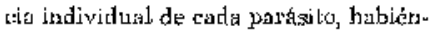
dose encontrado que los tres mis frocuentes son: la Uneinaria, el Ascaris y el Tricocéfalo.

Si se analiza, deside el punto de vista de la procedencia, de las tres ciudardes más importantes de ba Provincia ('Tabla III) se encuentra que nuhag diferencia apreciublo de frecuemria lelativa de puráisitos.

Manuifestaciones clinicas frecucutexEn primer lugar, no se ha encontrado sintomatología lipica para eadi parásito, pere aún, paral bas poliparasitosis. En sogundo lugar, por no ker uno sslo el ártano utacado por la parasitosis, 
TAIBLA I

RESULTADOS POSTTIVOZ $\mathrm{Y}$ NEGATTVOS OBTENIDAS EX

COPROPARASHARIOS EFECTUADOS EN LA CUUDAW DF GUARILUA, 1957

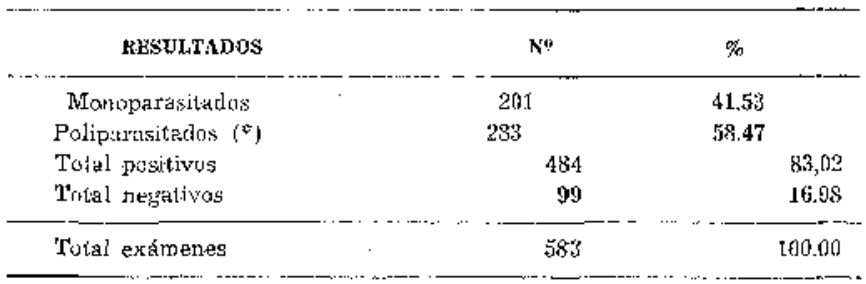

(*) La asuciación más frecuente en lus casus de poliparasitismú lie la de Uncinaria-Ascuris-Tricucéfalo: 225 wasos - $79 \%$ del total de puslipurasitados.

TABLA Il

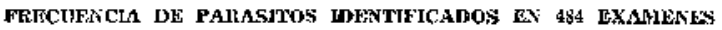

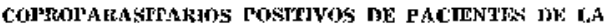
CIUDAD RE GLAKANDA, 1957

\begin{tabular}{|c|c|c|}
\hline PARASITYS & TOMAI & PORCENTAJI \\
\hline Necator americanuss & 231 & 47,72 \\
\hline Atrajis Iumbricnides & 211 & 44 \\
\hline Triehuris trichiura & 176 & 36,36 \\
\hline Eudiamocise histolytica & 71 & 14,66 \\
\hline Endamoeba coli & 52 & 10,74 \\
\hline Trichomomas intestinalis & 27 & 5,57 \\
\hline RaJantidium roli & 9 & 1,85 \\
\hline Tenia saginata & 7 & 1,44 \\
\hline Sirongylo:des stercolaris & 6 & 1,23 \\
\hline Chilomastix mesnilli & 4 & $\pi, 17$ \\
\hline Giarslia ]amblia & 3 & 0,61 \\
\hline Tenia sulium & 2 & 0,41 \\
\hline Opistorchisi & 1 & 0,20 \\
\hline Hymenolepis nana & $i$ & 0,20 \\
\hline
\end{tabular}




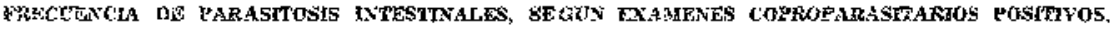
EN PACLENTES DE TRES CANTONBS DE LA PROVLNCLA DE HOLNAH, 1957

\begin{tabular}{|c|c|c|c|c|c|c|}
\hline \multirow[t]{2}{*}{ P.RASTEO } & \multicolumn{2}{|c|}{ c. DE GOARANDA } & \multicolumn{2}{|c|}{ c. Chillition } & \multicolumn{2}{|c|}{ C. SAN MHGURL } \\
\hline & $\begin{array}{l}\text { Exápuenes } \\
\text { Pesínives }\end{array}$ & Pumbintaje & $\begin{array}{l}\text { Exximenes } \\
\text { porzitatess }\end{array}$ & Porcentale & $\begin{array}{l}\text { Exúmedes } \\
\text { positivos }\end{array}$ & Porcertijaje \\
\hline Astarias lumbricoides & 125 & 27,24 & 42 & 22,32 & 44 & 28,57 \\
\hline Uncinaxia & 112 & 24,40 & $T \frac{1}{2}$ & 32,36 & 45 & 29,22 \\
\hline Trichuris trichiur. & 93 & 20,26 & 43 & $2 \overline{2}, 84$ & 10 & 25,97 \\
\hline Trichomonas intestinalis & 23 & 5,01 & 2 & 1,06 & 2 & 1,29 \\
\hline E. Hist. y E. coli. & 81 & 17,69 & 24 & 12,76 & 18 & 1168 \\
\hline Strongyloides stercolaris & 3 & 1,65 & 1 & 0,47 & 2 & 1,29 \\
\hline Balantidium coli & 4 & 0,82 & 2 & 1,06 & 3 & 2,59 \\
\hline Tenia solium & 2 & 0,43 & & & & \\
\hline Tenie sagunata & $\vec{\jmath}$ &., 56 & & & & \\
\hline Chilomastix mesnilli & 4 & 0,82 & & & & \\
\hline Opistorchis & 1 & 0,21 & & & & \\
\hline Gisardia lamblia & 3 & 0,65 & & & & \\
\hline Fiyulenolepis nasa. & 1 & 0.21 & & & & \\
\hline Total exámenes positivos: & 459 & 100,00 & 188 & 100,00 & 154 & 100,00 \\
\hline
\end{tabular}


siro varios, oncontramos un predonirisi de sintomas do acuerdo al órgano "l ótgasios más afectados.

Entre nisntros, es de muly frecuente observación, la compleja asocjación de buliparasitosis tropieal con paludismo $y$, on algunos edsos, tho daros por ciert $t$ con la tuberculosis.

Cumo síntomas, que más nos huth Jamad; fa atencinn en muestras pacien. tes, podemas citar los siguientes:

Síndrome carencial.-Es al sindrome que predomina, especialmente en la poliparasitosis tropical, elyo componente principal es al mnquilostcrina. Observamos que la "curva ponderal $y$ estatural se encuentra retardada". Esto obedces, a nuestro juicio, a múltiples factores: anorexia, alinuentación no balsursteada, etc. La piel del pardsitado ad. quiere un culor armarillo pílido, se vuelve sect; con pequeñas descamaciones lambjór amâri]lentas. El cabello se presenta deslustrado $\mathrm{y}$ con luna implanta. ción rula: tı hemos encertinato alopacia.

Iú facies está abotagada, especialmente en la región parotidia. Desaparece el parrículo adiposo an los juiembres, cuello y lónx. El thdomen se presenta abombado. Hay ligero edema palpebral $y$ on los miembrus, aunque en alşıนnos rasos, hemos tenido verdadercis andsirtis. En pacientes cun pir liparesitosis trotjical asociada a paludismo se han identificado manifestaeinnes elfuicas de glomérulonefritis, comprobara can los datos do laboratoris.

En la lengua se observa atrofía parpilaı y edema, en el que dejan huellas los piozas dentiarias.
Hemos encontrads también ligeras hopatorregalias, sobre tudo en las urieturtriasis, Frn ceasi todos los sasos, el bazo as percutible.

Síndrome gasirointestinal.-Torla la patologia del aparato kigestivo, podriamiss relicicurarta con las pau'asitusis irltestinalas; entre las manifestaciones rzás comines que hernos encostrado tenemus:

Anorexia (cosi en el 95\%), geofagia, núlusen, virnito especialmente alimenticio (strbre tads, en los niñas).

Dolior ubdoninal, siendo más frọcuente c] dolur difuso que el Jocalizado. Cuэndo es localizado, el dolor mas frecuente es ol peritumbilical, hego a nive! de la fosa iliaca derecha; $y$, en alcunns ('as)s, se el cpigatrio.

Diarrea, que puede ser acuosa, niumost, muco-singujnolenta, mucu- pu=ulenti. Se observa, sobre todo, en la atmbbinsis intestinal, en ba cual adquiere carácter srúnieo, acompañada siempre de molestids alytorimales y tenesmo.

Fin loz niños, ess tspecialrnente el A.بcaris lumbricoides el que provoca esti diarrea sróníca, la mísma que desupawase tars prorth se justiluye el tratamiento correspondiente. Es de obser. vación frecuebte el Prolapso rectal.

Sisiena nervioso.-Por parte del sistemil nerviuso, encontramos: irritabilidad, instmnio, prur:to anal y naзat, castuňe:eo de dientes, sialurrea. En oedsienss convulsiones tónjecr-chónicas.

Aparate respiratozis.-IJemos cnechltrade: bronconeumonía, bronquitis ilguda ismatiforme, focos bronconeumónievs diruusteritos. En el laburatorio en torlos ostos easos, existo eosinofilia. 
Dan muchas veoes manifestationes de neurionitis bacteriana.,

Én 4 ó 5 casosi hemos encoustrado la "Nefrit is hidropigena" de que nos hiblan Tanea Marengo y Francisco R(1jas, que ess or'jginada, segím ellus, por unia "tuxina retrolóxić, proxlueida por el anquilestoma $y$ que nis iffecta protuadaruter al epitelio rendt". Esto la aseguramo, prrque una vez olimirados Los parásitos con el tratamiento respectivo, desajarecicron los trastormos renules; la cual se comprobó eor el examen de laburatorio.

En casí toctus les casun, existen manifestaciones de hipoproteincuia.

AneDia.-Fin Juestras mobrvaciones, el numets de eritrocitos oscila centre

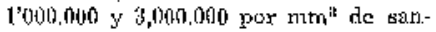
gro; sobre todo en la uncinar:asjis. Lst anerriat más frecuente es la microcí1.ica-hipucrómica (tipo da anema se. cundaria).

En las anemias e hipoproteinemias avanzidas, observarnos taquicordia: acertuducirin do los ruidos cardíacos, soplos plunifneales y hasia inmuficiencia circulatoria.

Eosinofilia- $N o$ es eorstante en todos los rasos. Pero hemos encontrudo exyinofilias del 5,10 y hasta del $25 \%$, que la deservé en un niño potipurasitado proveniente del subtrópico.

\section{IRESUMEN}

EII problema de las parasitosis intostinales en nucstro pais y, especialmente en ]al Provinaria de Holivar, basta hoy no hir podido ser debidamente estudiado ni resuelto.
Es posible que los jndices de morbilidad puedan ser modificador, auncue en mínima parte, mediante une invest.igusión olínica orientiada cu forma sistemática it descubrir poribles ixifestaciones parusiterias el todos nuestros pareicenter.

A pester de que nit existe una sintomatoloría curacterítica para cadu parúsito, sin ermbargo el sirtirme carencial y ricrtos trastornos gastruin lestinales que no obedecen a una causa perfeclamerte identificada, pueden hacer susperhar nuna inlestasiớn parasitaria.

De un tertal de $\mathbf{5 8 3}$ exámunes coproporasitarios efectuados, se ha obtenisdo $83,02 \%$ de resustados positivos; de éstos, más de la mitad $(58,47 \%)$ fucron. poliparasitados.

Los parásitos más frecuentemente identificados fueron cot brden decre ciente: Lncinnria $47,72 \%$; Ascaris bricoides $44 \%$; Trichuris trichura $36 \%$; Endamueba histulytica $14,66 \%$. Los restantes aparecen en proporción inculor.

Se incluyen además resultados partriales de 3 cantones de la Provineia de Bolívar.

\section{SUMMARX}

The problen of intestinul parasites in our country, especially in the province of Bolivar, up to now, has not been properly studien or resolved.

It is prossibie that the inder of morbidily ean be modified slightry by means of a well sriested clinical jnvestigation and individuad treatment in ajl patiants. 
There is ne characteristic sy:upiomology for cach parasite, there are malnutufion atid certain gastro-inteslind upsets that cannut be aftributed lo a cortain ceruse and leads to a suspicion of parasitic infestations.

From 583 examinatisin we have hat $87.52 \%$ positive to intestinal parrsites and more than hall presented several parasites.

The nust frocuently identified were as follows: Uncinuria, $47.72 \%$; Ascaris Jumbricoides, $44 \%$; Trichnis txichura, $36 \%$; Lndanoeba listolytica, $14.56 \%$.

'The others ocurped less Ireguentuty.

Included are other particil results in three Carixims of the Province of Bolivar.

\section{CLASIFICACHON DE VIRUS}

la mayoria de los cuales producen lesiones cutáneas

\section{(E)'บ1)}

Vitus Pox:

Viuloloidos

Virusla

Fistomatitis vesiouloxy oviri,

Nonluscus contioyiosum

Núdulos ade los lecheros

Vitus berpéċicos:

$\operatorname{sinpl}$

Simio (B)

Varictly-Zoster

Visus cítomegálicos (glúndukas solivares):

Mixovidus:

Infítuenza A, B y C

Parainfluen 1, 2, 3 y 4

$\mathbf{p}_{\text {iाpw }}$

Enfermadad de New-Castke

Sitrmpion

ATborin'us:

Gtippo A, B, C, ck:

Picomonvirus:

Pyliomie!itis

Coxackia

FCHO

Rimovisus

Adenorieus

papjlana-puitoma

Yerrugas

Rocosla spidémica

Reovirus
T'annairo Acido nurleico (milinierones)

$150-300$

DNA

$130-2[4$

$230-310$

120-2 -

$100)-200$

DNA

DNA

BQ-150 RNA

$20.7,00$ HNA

$20-30 \quad$ RNA

$\begin{array}{ll}70 & \text { DNA } \\ 25-45 & \text { DNA } \\ 35-55 & \text { DNA } \\ 100-300 & \text { RNA } \\ 70 & \text { RNA }\end{array}$

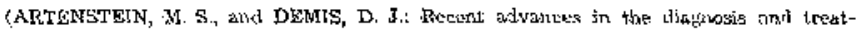

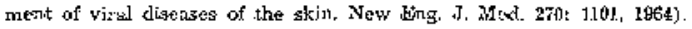

